\title{
Investigation of Zigzag Rising Bubble Using Spinning Sphere Model*
}

\author{
Masamichi NAKAGAWA**, Masanori ARITOMI***, \\ Yumiko KUMANO ${ }^{* * * *}$ and Michitsugu $\mathrm{MORI}^{\dagger}$
}

\begin{abstract}
A zigzag rising motion is a typical motion of bubbles in bubbly flows. Vortices shedding oscillatory behind a bubble and the wake of the bubble turning periodically similar to a sign of inequality are regarded as the most fundamental causes of the turbulence excited in bubbly flows, because these phenomena produce an alternating lift force on the basis of both conservation laws of circulation and momentum, respectively. In this paper, we discuss the lift production on a zigzag rising bubble trailing a hairpin vortex using a spinning sphere model, which is constructed by us to hold the aerodynamic universality independent of Reynolds number. We fluid-dynamically explicate the alternating lift production on a rising originally nonspin bubble and the zigzag turning of its path, and propose a chain of processes considered in this paper as a fluid-dynamic mechanism that produces the rising bubble zigzag motion.
\end{abstract}

Key Words: Bubble, Zigzag Rise, Hairpin Vortex, Lift, Added Mass

\section{Introduction}

The velocity profiles around bubbles in bubbly flows are measured using an ultrasonic velocity profile monitor (UVP) within the bubble Reynolds number limits of $400<R e_{\mathrm{B}}<1200$, and the existence of a boundary layer around each bubble and its characteristics are elucidated $^{(1)-(8)}$. The bubble boundary layer exhibits a transition from laminar to turbulent. Critical bubble Reynolds numbers are $R e_{\mathrm{B}, \text { crit }} \approx 1000$ without the effect of leading bubbles and $R e_{\mathrm{B}, \mathrm{crit}}=600-750$ with the effect of leading bubbles. In the bubble Reynolds number range considered, zigzag rising bubbles are observed in bubbly flows.

A zigzag rising motion is a typical motion of bubbles in bubbly flows. Vortices shedding oscillatory behind a bubble and the wake of bubble periodically turning simi-

* Received 20th March, 2006 (No. 06-4065)

** Department of Mechanical Science and Engineering, Tokyo Institute of Technology, 2-12-1-I3-3 Ohokayama, Meguro-ku, Tokyo 152-8552, Japan.

E-mail: nakagawa@mech.titech.ac.jp

*** Laboratories for Nuclear Reactors, Tokyo Institute of Technology, 2-12-1-N1-13 Ohokayama, Meguro-ku, Tokyo 152-8550, Japan

**** Tokyo Electric Power Company, 1-1-3 Uchisaiwai-cho, Chiyoda-ku, Tokyo 100-0011, Japan

† Tokyo Electric Power Company, 4-1 Egasaki-cho, Tsurumi-ku, Yokohama 230-8510, Japan lar to a sign of inequality are regarded as the most fundamental causes of the turbulence excited in bubbly flows, because these phenomena produce an alternating lift force on the basis of both conservation laws of circulation and momentum, respectively ${ }^{(3),(7)}$.

As well known in the turbulent flow of a single phase, the vortices over wideband scales form coherent structures and their behaviors govern turbulence characteristics. Accordingly, also in the bubbly flow that is one of the flow patterns representing multiphase flows, it is very important to consider vortex structures for clarifying turbulences.

A concept for the lift production on a bubble had already been described ${ }^{(3)}$ : The boundary layer as a vortex sheet separated from the bubble and formed a starting vortex that induced circulation. Because the reverse of such circulation remained on the originally nonspin bubble, the bubble held the circulation. Consequently, a lift was produced on the bubble. However, the direction and magnitude of the lift were not presented concretely and quantitatively.

It is necessary to consider not only the motions of visible vortices around a bubble but also the dynamics of the entire vortex structure including the bubble that is itself a vortex (i.e., bound vortex) for explicating complex motions with the rocking and deformation of zigzag rising bubbles.

In this study, a spinning sphere model, which is constructed to hold the aerodynamic universality with- 
out Reynolds number dependence, is applied to a hairpin vortex $^{(11),(12)}$ observable as an elementary structure in the wake of a bubble. A theoretical framework useful as a template for analyzing and verifying the results of experimental measurements and numerical simulations, and as a reference model for instrument and control is proposed for bubbles in zigzag rising motion.

\section{Aerodynamics of Spheres in Spin}

At the beginning of this section, we restrict our discussion to circular cylinders and spheres whose coefficients of moment can be neglected, because of their reducibility for the redundant grade of freedom among three coefficients of aerodynamics (i.e., drag, lift and moment coefficients).

\subsection{Principles of lift production}

2.1.1 Kutta-Joukowski's theorem The circulation $\Gamma$ around a body in a potential flow (i.e., inviscid and irrotational) of density $\rho$ and uniform flow velocity $U$ produces a lift per unit span, $L=\rho U \Gamma$. However, a cylinder or a sphere rotates in the inviscid potential flow, no circulation is generated in the inviscid fluid around the rotating body, and also no lift is produced, because the flow slips perfectly on the surface.

2.1.2 Magnus effect In the case of the rotation of a body in a real viscous flow, when the Reynolds number $R e$ is not sufficiently larger than the order of 1 , the fluid around the body dragged by the surface of the rotating body produces the circulation $\Gamma$ and the lift per unit span $L=\rho U \Gamma$, because the surface of the body satisfies nonslip conditions. However, the actually produced lift decreases owing to a certain loss.

In contrast, when the Reynolds number is larger than the order of 1 , the lift production on a cylinder or a sphere rotating in a real viscous flow is not simply explicable as the Magnus effect, because the fluid flow can not adhere closely to the surface. For example, behind a nonspin cylinder, the twin vortices are attached at $R e>5$ and the Kármán vortex street is observed at $R e>46$.

2.1.3 Asymmetry of boundary layer separation Specifically for a high Reynolds number of actual importance $(R e>1000)$, we consider that the asymmetry between the top and bottom separations of the boundary layer around a body determines the positive or negative sign and magnitude of the produced lift.

That is, on the top of a back-spinning sphere, the slow development of the laminar boundary layer makes the late transition to turbulent boundary layer, because the relative velocity between the circumferential velocity $u_{\theta}$ and the uniform flow velocity $U-u_{\theta}=U\left(1-u_{\theta} / U\right)$ is smaller than $U$. Oppositely, at the bottom of the back-spinning sphere, the fast development of the laminar boundary layer makes the early transition to turbulent boundary layer, because the relative velocity between the circumferential velocity $u_{\theta}$ and the uniform flow velocity $U+u_{\theta}=U\left(1+u_{\theta} / U\right)$ is larger than $U$.

After these processes, an asymmetry occurs, in which the top side of the back-spinning sphere exhibits laminar separation, and its bottom side, turbulent separation, the wake turns upwash and the reaction produces a negative lift $^{(9),(13)}$, because the downstream distance to the turbulent separation position is longer than that to the laminar separation position. Such a negative lift production inexplicable by the Magnus effect vividly demonstrates the importance of the top-and-bottom asymmetry of boundary layer separation.

\subsection{Analysis of lift production by downwash}

2.2.1 Flow modeling around back-spinning sphere It is necessary for a uniform flow past a back-spinning sphere to be modeled as a three-dimensional flow (Fig. 1) owing to the finite span of the sphere. Because a backspinning sphere producing a lift has a low pressure on the top side surface and a high pressure on the bottom side surface, the flows climbing from bottom to top on both flanks of the sphere are induced. These induced flows move at the uniform velocity $U$ toward the downstream behind the sphere. Therefore, they start from both backspin axis poles on both sphere flanks and grow into a pair of longitudinal vortices rotating inversely each other toward the downstream behind the sphere at the length per unit time (i.e., the uniform velocity) $U$.

By modeling the back-spinning sphere as a fixed vortex (i.e., bound vortex) that holds the circulation around the back-spin axis $\Gamma$ shown in Fig. 2, we can develop a model in which Helmholtz's vortex theorem (i.e., continuity of a vortex filament) and Kelvin's circulation theorem (i.e., conservation law of circulation in a perfect fluid) produce the circulation of the longitudinal vortices $\Gamma$ mentioned above (i.e., trailing vortices); both vortex terminals are connected with a starting vortex with the circulation $\Gamma$ closing as a vortex ring with the circulation $\Gamma$ in its entirety. Then a downwash velocity, $w$, is induced inside the vortex ring behind the back-spinning sphere.

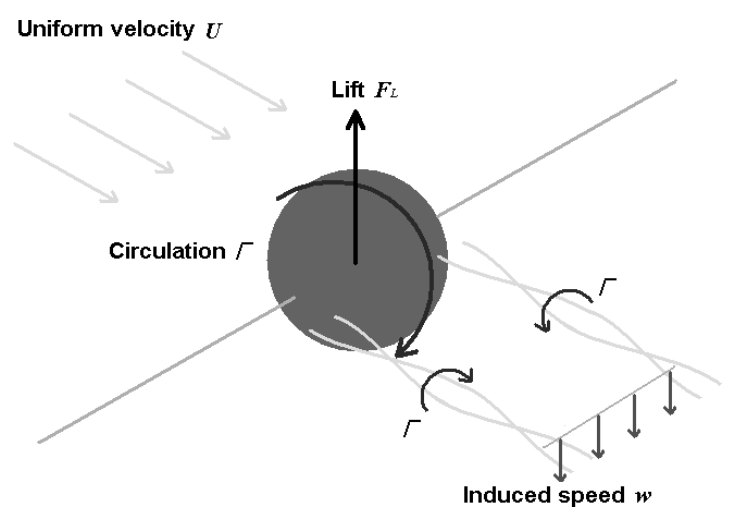

Fig. 1 Aerodynamic model of sphere back-spinning in uniform flow. 


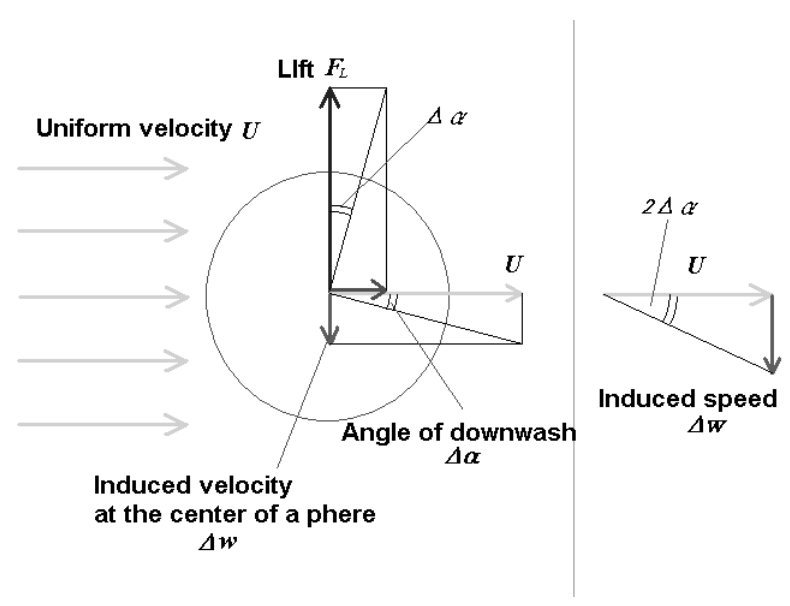

Fig. 2 Geometric relation concerning downwash at center of back-spinning sphere.

2.2.2 Lift and law of momentum The correlation between the produced lift $F_{\mathrm{L}}$ and the induced velocity $w$ is written on the basis of the law of momentum as

$$
F_{\mathrm{L}}=(\rho U A) w,
$$

where $A$ is the projection area of the sphere $\left(\pi D^{2} / 4\right)$ and $D$ is the diameter of the sphere.

There is a correspondence of

$$
\int \Gamma d z=A w
$$

where $z$ is the coordinate in the direction of the span, between Eq. (1) and Kutta-Joukowski's theorem, which is essentially two-dimensional. In real viscous fluids, because the circulation $\Gamma$ is not conserved by damping due to molecular and eddy viscosities, the induced velocity $w$ decreases. Therefore, the produced lift $F_{\mathrm{L}}$ also decreases as mentioned in subsection 2.1.2.

2.2.3 Induced drag and energy balance The mass per unit time of the fluid $\rho U A$ with the abovementioned induced velocity $w$ has the kinematic energy of downwash per unit time $\frac{1}{2}(\rho U A) w^{2}$. Because the source supplying this kinematic energy is only the back-spinning sphere in the flow field of the uniform velocity $U$, the induced drag corresponding to the above-mentioned energy $F_{\mathrm{Di}}$ is produced as the component of the lift $F_{\mathrm{L}}$ and then the energy per unit time $F_{\mathrm{Di}} U$ is appropriated from the flow of the uniform velocity $U$ for the kinematic energy of downwash.

In other words, the work per unit time (i.e., power) $F_{\mathrm{Di}} U$ for maintaining the flow field of the uniform velocity $U$ against the induced drag $F_{\mathrm{Di}}$ is supplied as the energy per unit time for the back-spinning sphere to generate the pair of trailing vortices mentioned in subsection 2.2.1. Moreover, it is transformed into the kinematic energy of downwash mentioned above. Hence, the energy balance of the downwash flow per unit time is

$$
F_{\mathrm{Di}} U=\frac{1}{2}(\rho U A) w^{2},
$$

where the energy loss is neglected in order to restrict our discussion to the fluid dynamical principle.

2.2.4 Correlation between lift and induced drag Substituting Eq. (1) into Eq. (2), the correlation between the induced drag and the lift is obtained as

$$
F_{\mathrm{Di}} U=\frac{1}{2} F_{\mathrm{L}} w \quad \therefore F_{\mathrm{Di}}=F_{\mathrm{L}} w /(2 U) \text {. }
$$

Whereas two-dimensional potential flows do not produce drag in accordance to D'Alembert's paradox, threedimensional flows including a circulation of finite span are characterized by the occurrence of the induced drag $F_{\mathrm{Di}}$ not to be viscosity-derived, under the production of the lift $F_{\mathrm{L}}$, shown in Eq. (3).

By eliminating the induced velocity $w$ from Eq. (3) in combination with Eq. (1), the induced drag $F_{\mathrm{Di}}$ is written as

$$
F_{\mathrm{Di}}=F_{\mathrm{L}}^{2} /(2 U \cdot \rho U A)=F_{\mathrm{L}}^{2} /\left(4 \cdot \frac{1}{2} \rho U^{2} A\right) .
$$

Furthermore, using the definitions of both the coefficients of induced drag and drag, we obtain the next correlation

$$
C_{\mathrm{Di}}=C_{\mathrm{L}}^{2} / 4 \text {. }
$$

2.2.5 Correlation between downwash angle and lift coefficient The correlation between the induced velocity of downwash $\Delta w$ and the angle of downwash $\Delta \alpha$ (i.e., induced angle of attack) at the center of the back-spinning sphere satisfies the following equation for $\Delta \alpha \ll 1$,

$\Delta w / U=\tan \Delta \alpha \approx \Delta \alpha$.

By considering $\Delta w$ as the arithmetic mean between the induced velocity of downwash in front of the backspinning sphere to be 0 and the induced velocity of downwash behind the back-spinning sphere $w$,

$$
\Delta w=w / 2 \text {. }
$$

By substituting Eq. (6) into Eq. (5), and arranging the substituted Eq. (5) by Eqs. (3) to (4) in order, we can obtain

$$
\begin{aligned}
& \Delta \alpha=\Delta w / U=w / 2 U=F_{\text {Di }} / F_{\mathrm{L}}=C_{\mathrm{Di}} / C_{\mathrm{L}}=C_{\mathrm{L}} / 4, \\
& \therefore C_{\mathrm{L}}=4 \Delta \alpha .
\end{aligned}
$$

In real viscous fluids, it is assumed that the proportional coefficient 4 in Eq. (7) decreases considerably owing to the various losses mentioned at the later parts of subsections 2.1.2, 2.2.2 and 2.2.3.

2.2.6 Spin parameter It is considered that the parameter concerning the lift production of the backspinning sphere is the ratio of circumferential velocity to the uniform velocity $u_{\theta} / U$ based on the principle mentioned in section 2.1. Because the circumferential velocity on the surface of the back-spinning sphere $u_{\theta}$ is considered as the relation of the back-spin rate $n$ and the next equation holds good

$$
u_{\theta}=\pi D\left(n_{\mathrm{rpm}} / 60\right)=\pi D n,
$$


where $n_{\mathrm{rpm}}$ is the back-spin rate per minute [rpm] and $n$ is the back-spin rate $[1 / \mathrm{s}]$, the ratio of circumferential velocity to uniform velocity is written as

$$
u_{\theta} / U=\pi D n_{\mathrm{rpm}} /(60 U)=\pi D n / U .
$$

\section{Dynamics of Bubble and Hairpin Vortex}

We choose to discuss the fluid dynamics for explicating the zigzag motion of a rising bubble qualitatively in this paper before quantitatively in future works.

\subsection{Hairpin vortex as wake of bubble}

3.1.1 Experimentally observed results The hairpin vortex has been observed as the wake behind a solid sphere by Möller ${ }^{(10)}$ and behind a bubble by Lunde and Perkins ${ }^{(11)}$ (reproduced by Brücker ${ }^{(12)}$ : see Appendix Fig. 1) about the order of our experimental bubble Reynolds number range, $400<R e_{\mathrm{B}}<1200$. This is a U-type vortex in which the head corresponds to a starting vortex and the legs correspond to a pair of trailing vortices from the sphere or bubble. By collation with the spinning sphere model discussed in section 2, the hairpin vortex is considered as one of the elementary structures of vortices in the wakes behind spheres and bubbles.

From this standpoint, we redefine the following two phases observed in the image of the hairpin vortex (Fig. 6 of Brücker $\left.{ }^{(12)}\right)$ by Lunde and Perkins ${ }^{(11)}$.

Zig phase: At first, the hairpin vortex of the wake behind the bubble extends from the upper right to the lower left. This shows that the bubble rises almost straight in right ascent during this "zig" phase, advancing about 5 times the bubble diameter. Therefore, it becomes evident that the negative lift corresponding to the top spin acts almost constantly on the bubble during this phase.

Zag phase: Also, the bubble turns to the upper left. This shows that the sudden force toward the upper left acts on the bubble at the terminal of the zig phase and that the path of the bubble turns around suddenly to the upper left. Consequently, it is determined that the force corresponding to the positive lift acts on the bubble and that the phase changes to "zag" phase.

After changing to the zag phase, by turning over between right and left, the phase can be regarded as the zig phase again where the negative lift corresponding to the top spin acts almost constantly on the bubble.

\subsubsection{Steady and transient modes By arrang-} ing the zigzag rising motion of the bubble from the viewpoint of the spinning sphere model in section 2, we observe that the zigzag rising motion consists of two elementary modes as follows:

( a ) In the steady straight-advance mode, the negative lift corresponding to the top spin acts almost constantly on the bubble trailing a hairpin vortex.

(b ) In the transient turning mode, the sudden force corresponding to the positive lift acts on the bubble and turns its path suddenly similar to a sign of inequality at the terminal of the steady straight-advance mode (a).

However, recent studies seem to advance no theory based on fluid dynamics exactly for even such fundamental characteristics as the direction and magnitude of the lift acting on a bubble trailing a hairpin vortex.

In section 3.2, we elucidate these fundamental characteristics of fluid dynamics by applying the spinning sphere model discussed in section 2 to the vortex ring of a bubble and a hairpin vortex.

\subsection{Application of spinning sphere model}

3.2.1 Vortex ring of bubble and hairpin vortex By considering the relation between a zigzag rising bubble and a hairpin vortex, because the hairpin vortex of a filament does not have an end in the fluid (i.e., liquid phase) within the three-dimensional flow field due to the conservation law of circulation, the hairpin vortex satisfies either of the two cases as follows:

(i ) the generation and extinguishing of the hairpin vortex filament on the bubble surface as the interface between the different phases and

(ii ) the closure of the hairpin vortex filament as a vortex ring in the liquid phase.

The standing of the legs of the hairpin vortex directly on the bubble surface (i.e., gas-liquid interface) in case (i), is a very unreasonable fluid-dynamic characteristic of the zigzag rising bubble without a forced spin.

In contrast, in case (ii), it is considered that the bubble is covered with the boundary layer of the vortex sheet from the front stagnation point and that the boundary layer separates asymmetrically getting the circulation to connect with the hairpin vortex as its legs. Hence, this is a very natural fluid dynamical characteristic.

Consequently, we recognize the formation of a vortex ring three-dimensionally composed of a bubble boundary layer and a hairpin vortex, because the hairpin vortex can be closed as a vortex ring in the liquid phase by connecting its legs with a pair of trailing vortices extending from the bubble boundary layer.

3.2.2 Top-spinning circulation around bubble By referring to the result obtained by Lunde and Perkins $^{(11)}$ (Fig. 6 of Brücker ${ }^{(12)}$ ), the circulation held in a section around the vortex ring of the bubble boundary layer and the hairpin vortex proves to be always in the top spin around the bubble as the steady straight-advance mode (a) in subsection 3.1.2.

Although hairpin vortices with alternate exposures are succeeding one's head after another's legs in the vortex chain $^{(12)}$ (see Appendix Fig. 2) as the wake of the bubble, resolving them into units of the steady straight-advance mode (a) in subsection 3.1.2 and applying the spinning sphere model of section 2 into each unit of the above steady straight-advance mode prove that the circulation around a bubble is always in the top spin. 


\subsubsection{Negative lift acting on bubble Accord-} ingly, the vortex ring of the boundary layer around a bubble and its hairpin vortex generally has always the circulation top-spinning around the bubble in the steady straightadvance mode mentioned in subsection 3.2.2. Hence, the wake always turns upwash and then the angle of downwash is negative $(\Delta \alpha<0)$ in Eq. (7), theoretically demonstrating that the negative lift always acts on a bubble almost constantly under the above-mentioned conditions.

This theoretical result agrees well with the results for the hairpin vortex obtained by Lunde and Perkins ${ }^{(11)}$ (Fig. 6 of Brücker ${ }^{(12)}$ ) concerning the quasi-steady inclination and transient turning of the bubble path similar to a sign of inequality and fluid-dynamically explains them very well.

Incidentally, the coefficient of 4 in Eq. (7) decreases owing to a certain loss and is affected by the asphericity of a bubble.

\subsection{Added mass effect of a bubble}

3.3.1 Failure of negative lift Why does the bubble change its direction to advance suddenly similar to the mode (b) of transient in subsection 3.1.2 from the observation, though the negative lift always acts on the bubble almost constantly according to subsection 3.2.3?

As the reason, we consider that the bubble breaks away from the vortex ring.

That is, as the bubble rises almost straight-obliquely under the action of the negative lift almost constantly, the hairpin vortex stretches its legs (i.e., trailing vortex pair), strengthens its vorticity due to its diameter decrease as a vortex filament and becomes unstable owing to deformation by self-induction. The zone of a free vortex surrounding its vortex filament core (i.e., the forced vortex domain) is damped viscidly.

As a hypothesis we consider that in the concurrence of the above-mentioned instability and damping of the hairpin vortex, the bubble within a short time sheds its boundary layer in asymmetric separation shouldering the circulation around itself, similar to the ecdysis of cicadas.

On the bubble, the top-spinning circulation vanishes suddenly and the negative lift having acted quasi-steadily also fails suddenly, resulting in a transient motion. Simultaneously, the added mass effect of the bubble appears in the horizontal direction in which the negative lift vanishes once transiently.

3.3.2 Superficially positive transient lift This indicates that the motion of a bubble in a liquid phase is governed by the added mass term because the density of gas is much smaller than that of liquid (i.e., $\rho_{\mathrm{g}} \ll \rho_{\mathrm{l}}$ ). With the approximation of the bubble to a sphere, neglecting both drag and Basset term, the horizontal component is described by

$$
\frac{1}{2} \rho_{1}(\pi / 6) D^{3} \times(\text { acceleration })=0-(\text { negative lift })
$$

$$
\begin{aligned}
& =0-\rho_{1} U \int(-\Gamma) d z \\
& =\rho_{1} U \int \Gamma d z .
\end{aligned}
$$

The last term in Eq. (10) represents the positive lift, and Eq. (10) indicates that the bubble behaves as if under the effect of the superficially positive transient lift.

When the negative lift vanishes suddenly in this manner, the lift superficially inverted to become positive with the same magnitude acts on the bubble transiently and reverses the horizontal motion of the bubble completely.

The above-mentioned processes explicate fluiddynamically explicate the alternating lift production on a rising originally nonspin bubble and the zigzag turning of its path. Hence, we propose such phenomena as the fluiddynamic mechanisms that produce a rising bubble zigzag motion.

\section{Conclusions}

Our conclusions drawn from the above discussion are as follows:

( 1 ) We theoretically analyzed the fundamental of the uniform flow past a back-spinning sphere by modeling a three-dimensional flow and proved the direct proportion between the angle of downwash and the coefficient of lift in the wake behind the sphere.

(2) From the observations by various authors, we considered that a bubble in a steady straight-advance mode forms a vortex ring connecting between its boundary layer and a hairpin vortex. We pointed out that the circulation of the vortex ring is always in the top spin around the bubble.

( 3 ) By applying the spinning sphere model of (1) to the vortex ring of (2), we theoretically demonstrated that the negative lift always acts on the bubble almost constantly because of always upwashing of the bubble wake.

(4) We presented a hypothesis in which the bubble within a short time sheds its boundary layer and breaks away from the vortex ring of (2), and in which concerning the bubble the top-spinning circulation vanishes suddenly and the negative lift also fails suddenly.

( 5 ) We demonstrated that the sudden failure of the negative lift due to (4) induces the action of the superficially positive transient lift on the bubble and its completely reversed motion in the horizontal direction.

(6) By the processes from (2) to (5), we fluiddynamically explicated the alternating lift production on a rising originally nonspin bubble and the zigzag turning of its path.

We propose a chain of processes summed up above for the fluid-dynamic mechanism that produces a rising bubble zigzag motion. 


\section{References}

( 1 ) Suzuki, Y., Nakagawa, M., Aritomi, M., Kikura, H. and Mori, M., Organized Multiphase Flow Forum '99 Yokohama, (in Japanese), (1999), pp.127-134.

( 2 ) Suzuki, Y., Nakagawa, M., Aritomi, M., Kikura, H. and Mori, M., Proc. 8th International Conference on Nuclear Engineering (ICONE-8), Baltimore, (2000), ICONE-8333.

( 3 ) Nakagawa, M., Suzuki, Y., Aritomi, M., Yabe, T. and Mori, M., Organized Multiphase Flow Forum 2000 Takayama, (in Japanese), (2000), pp.29-32.

(4) Suzuki, Y., Doctor Thesis at Tokyo Institute of Technology, (2001).

( 5 ) Nakagawa, M., Suzuki, Y., Aritomi, M., Yabe, T. and Mori, M., Proc. International Conference on Multiphase Flow (ICMF-2001), New Orleans, (2001), CDRNo.240.

( 6 ) Nakagawa, M., Kumano, Y., Aritomi, M. and Mori, M., Proc. FEDSM'03, Hawaii, (2003), FEDSM200345567.

( 7 ) Nakagawa, M., Suzuki, Y., Aritomi, M., Yabe, T. and Mori, M., Organized Multiphase Flow Forum 2001 Fukushima, (in Japanese), (2001), pp.1-2.

( 8 ) Nakagawa, M., Kumano, Y., Aritomi, M. and Mori, M., Proc. 11th International Conference on Nuclear Engineering (ICONE-11), Tokyo, (2003), ICONE1136503.

( 9 ) Nakagawa, M., Yabe, T., Misaki, M., Manome, K., Yamamoto, R., Tsugawa, Y. and Yamada, T., Proc. International Conference on Jets, Wakes, and Separated Flows (ICJWSF-2005), Toba, Japan, (2005), P-095.

(10) Möller, von W., Physik. Z., Vol.39 (1938), pp.57-80.

(11) Lunde, K. and Perkins, R.J., Proc. ASMEFED Summer Meeting, Vancouver, Canada, (1995), FEDSM973530.

(12) Brücker, C., Physics of Fluids, Vol.11 (1999), pp.17811796.

(13) Tritton, D.J., Physical Fluid Dynamics (2nd ed.), (1988), Chap.13, Oxford Univ. Press.

(14) Landau, L.D. and Lifshitz, E.M., Fluid Dynamics, (1959), §45, Pergamon, London.

\section{Appendix}
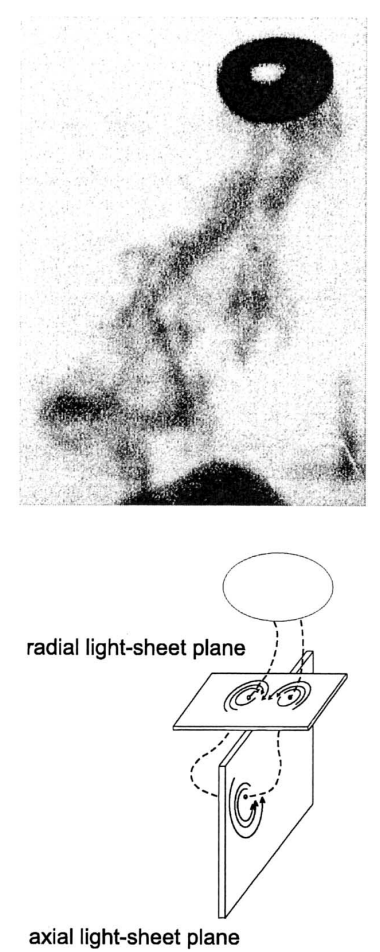

Appendix Fig. 1 Example of hairpin vortex. This figure is quoted from Fig. 6 in Ref. (12) by Brücker who considered the original visualization of Lunde and Perkins ${ }^{(11)}$.

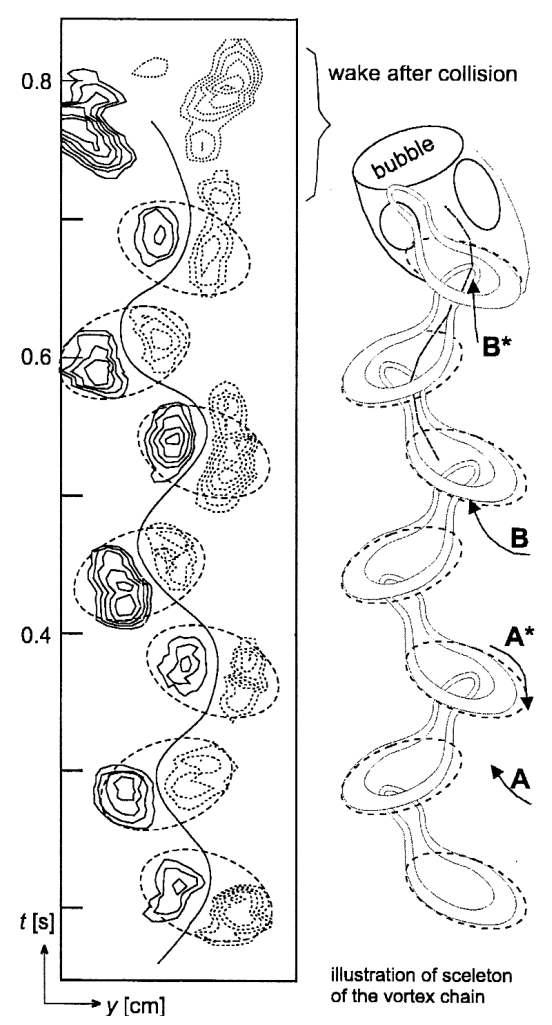

Appendix Fig. 2 Example of vortex chain. This figure is quoted from Fig. 10 in Ref. (12) by Brücker. 